\title{
ON THE INTERLACING OF CYLINDER FUNCTIONS
}

\section{T. PÁLMAI}

Abstract. Necessary and sufficient conditions for the interlacing of the zeros of cylinder functions and their derivatives of different orders are given.

Mathematics subject classification (2010): 33C10.

Keywords and phrases: Bessel functions, zeros of Bessel functions, interlacing.

\section{REFERENCES}

[1] M. Abramowitz And I. A. Stegun, Handbook of Mathematical Functions, Dover Publications, New York, 360-371, 1972.

[2] Á. ELBERT, Some recent results on the zeros of Bessel functions and orthogonal polynomials, J. Comp. Appl. Math. 133 (2001), 66-83.

[3] A. Laforgia And Pierpaolo Natalini, Zeros of Bessel functions: monotonicity, concavity, inequalities, Le Matematiche 62 (2007), 255-270.

[4] H. Y. LiU AND J. Zou, Zeros of the Bessel and spherical Bessel functions and their applications for uniqueness in inverse acoustic obstacle scattering, IMA J. Appl. Math. 72 (2007), 817-831.

[5] H. Y. LiU AND J. Zou, Zeros of the Bessel and spherical Bessel functions and their applications for uniqueness in inverse acoustic obstacle scattering problems, Technical Report CUHK-2007-02 (342), The Chinese University of Hong Kong, Hong Kong, 2007.

[6] T. PÁlmai And B. ApagYi, On nonsingular potentials of Cox-Thompson inversion scheme, J. Math. Phys. 51 (2010), 022114.

[7] T. PÁlmai And B. Apagyi, Interlacing of positive real zeros of Bessel functions, J. Math. Anal. Appl. 375 (2011), 320-322.

[8] J. SEGURA, Interlacing of the zeros of contiguous hypergeometric functions, Numer. Algor. 49 (2008), 387-407.

[9] G. N. WAtson, A treatise on the theory of Bessel functions, Cambridge University Press, 1944. 